\title{
Multimodal imaging techniques for the extraction of detailed geometrical and physiological information for use in multi-scale models of colorectal cancer and treatment of individual patients
}

JOE PITT-FRANCIS $\dagger^{*}$, DAN CHEN $\dagger$, MARK SLAYMAKER $\dagger$, ANDREW SIMPSON $\dagger$, MICHAEL BRADY $\$$, INGEBORG VAN LEEUWEN $\rrbracket$, FIONA REDDINGTON§, PHIL QUIRKE $\|$, GINA BROWN\# and DAVID GAVAGHAN $\dagger$

†Oxford University Computing Laboratory, Wolfson Building, Parks Road, Oxford OX1 3QD, UK $\ddagger$ Department of Engineering Science, Parks Road, Oxford OX1 3PJ, UK

I School of Mathematical Sciences, Centre for Mathematical Medicine, University of Nottingham, Nottingham NG7 2RD, UK

§NCRI Informatics Coordination Unit, 61 Lincoln's Inn Fields, London WC2A 3PX, UK

||Department of Pathology, Leeds General Infirmary, Leeds LS1 3EX, UK

\#Royal Marsden Hospital, Downs Road, Sutton, Surrey SM2 5PT, UK

(Received 1 June 2006; in final form 14 June 2006)

\begin{abstract}
A vast array of mathematical models have been proposed for all stages of cancer formation across a wide range of spatio-temporal scales. Attention is now turning to coupling these models across scales and building models of "virtual tumours" for use in in silico testing of novel drugs and treatment regimes. This leads naturally to the requirement for detailed knowledge of the underlying geometry and physiological properties of individual tumours for use in: (i) multi-scale mathematical models of in vivo tumour growth and development; (ii) fusion of multi-scale, multimodal medical imaging techniques to improve the diagnosis and treatment of individual patients; and (iii) training of cancer specialists and surgeons.
\end{abstract}

Keywords: Multimodal imaging; Colorectal cancer; Virtual tumors; Diagnosis

\section{Introduction}

Over the past 30 years, a large number of mathematical models of cancer have been developed that focus on different aspects of tumour growth, progression and treatment (see $[1,2]$ for comprehensive reviews). Such models inevitably cut across a wide range of spatialtemporal scales, as they quantify biological and physiological processes occurring at levels of organisation from the molecular, through the cellular and tissue levels, to the whole tumour, with its associated direct and metastatic effects on the host organism. Only recently has attention begun to turn to the problem of coupling such models, in order to build

*Corresponding author. Email: joe.pitt-francis@comlab.ox.ac.uk 
comprehensive, multi-scale "virtual tumours" for in silico testing of novel drugs and treatment regimes (see for example [3]). The multi-scale nature of the problem (in both space and time) has been neatly summarised by Bellomo and Maini [4] at the sub-cellular, cellular and macroscopic scales. Such multi-scale approaches, which ultimately aim to develop and validate detailed mathematical models within the heterogeneous in vivo environment, lead naturally to the requirement for detailed knowledge of the underlying geometry and physiological properties of individual tumours in situ for use in: (i) multi-scale mathematical models of in vivo tumour formation and growth; (ii) fusion of multi-scale, multimodal medical imaging techniques for the diagnosis and treatment of individual patients; and (iii) training of cancer specialists and surgeons. The goal of our research is a novel synthesis of mathematical and biological models of tumour formation and their validation using medical image analysis.

In this paper, we present our first steps towards developing some automated image analysis tools in the specific case of colorectal cancer. The paper is organised as follows. In the next section, we introduce the primary diagnosis and treatment approaches used for colorectal cancer, paying particular attention to the medical imaging and histopathological techniques that generate the input data for our automated tools. We then (Section 3) describe the image data available to us, which is derived from completed clinical trials carried out to evaluate the information potentially provided by magnetic resonance imaging (MRI) to assess the likely outcome of (total mesorectal) resection in patients with colorectal cancer. To date, our efforts (Section 4) have focused on developing and applying image processing and data registration techniques to reconstruct in silico the underlying in vivo anatomy of tumours from both in vivo MRI images and photographs and histopathology images of excised tissue samples. In Section 5, we give an overview of how we, as part of a larger team, intend to extend and use this data in building multi-scale mathematical models of tumour development and growth in a heterogeneous environment. For instance, we briefly summarise the specific goals in multiscale cancer modelling of the multi-institution Integrative Biology (IB) e-Science Pilot Project [5]. Furthermore, in Section 6, we discuss the IT infrastructure that we are currently developing and in particular, how it would enable our data extraction methods to be applied routinely and securely for individual patients in a clinical environment. Finally, we conclude with some remarks on the potential applications of our automated image analysis tools.

\section{The process of diagnosing and treating colorectal cancer}

Colorectal cancer is first diagnosed using endoscopy and confirmed by performing a biopsy. The cancer is then staged radiologically in order to determine the extent of local and distant lesions.

\subsection{An overview of diagnosis and treatment}

In this section, we follow the steps of a patient suffering from colorectal cancer. First, the patient, feeling discomfort, visits his/her doctor. The initial diagnosis will be based on the presence of one or more symptoms such as: altered bowel habits, rectal bleeding, weight loss, anaemia and a family history of colorectal cancer. Generally, the doctor either reassures the patient or refers him/her to a local hospital for more detailed examinations. Typically, the large bowel is examined and the diagnosis confirmed using an endoscope. During the endoscopy procedure, the oncologist may take a biopsy. Increasingly, the patient is then sent 
to a radiologist to have a computed tomography (CT) or MRI scan taken. The former is a reconstructed 3D X-ray image of the region of interest, whereas the latter is a 3D image based on magnetic excitation in differing tissue types. These images can reveal some suspicious dense areas that might imply the presence of a tumour and can also provide early evidence of metastasis in the liver. Investigation for the presence of secondary tumours may also involve conducting a further scan, using CT, positron emission tomography (PET) or ultrasound, of the liver or lungs.

Based on the images, the tumour is staged using the TNM system (which takes account of tumour size, the number of sentinel lymph nodes in the mesorectal fascia deemed to contain malignant cells, and the likely presence of metastasis). Depending on the stage of the disease and on the patient's individual characteristics, a different patient management decision will be made. Among the possibilities are:

(i) The patient is sent for MRI scans, which provide information that is complementary to that provided by CT-primarily contrast between different soft tissues. The MRI scans are used to define the precise local extent of tumour spread and to identify other cancerous areas within the pelvis.

(ii) The patient proceeds to surgery. During this procedure, the surgeon generally "resects" (cuts out) the whole of the mesorectal region surrounding the tumour on the basis of the information provided by the CT (and increasingly, MRI) scans.

(iii) The patient is given a neoadjuvant course of radiotherapy or chemotherapy which typically lasts six weeks with the primary aim of downstaging the tumour (e.g. by reducing the risk of metastasis, reducing the number of affected nodes, and reducing the size of the tumour) particularly in cases where the tumour extends to the potential surgical excision planes. By achieving tumour regression prior to surgery the chances of a curative operation successfully taking place are increased.

(iv) The patient is sent to "palliative care". This is the case for patients whose tumours have reached a stage too advanced to be cured and for patients who are judged too weak to undergo therapy (for example, high-intensity focussed ultrasound) or surgery.

Survival rates after treatment show that there is a $95 \%$ probability of surviving 5 years if the cancer staging indicates that the disease is confined to the intestinal mucosa. However, this survival rate drops to $65 \%$ if the malignancy has invaded the bowel wall, to $30 \%$ if is has spread to regional lymph nodes and to $15 \%$ if distance metastases are present.

It is important to note that although that MRI images may have been taken prior to therapy, a second set of MRI scans may be taken prior to surgery (for example, to assess the effect of chemo/radiotherapy and to downstage the tumour) and even a third set post-operatively to assess the effect of surgery. If the tumour is resected (together with some of the surrounding colon), then further data may be recovered: high-resolution digital histopathology slides may be obtained from slices through the tissue and/or coarse sections may be photographed using a conventional digital camera.

\subsection{Tumour staging}

Since tumour staging is based on decisions that involve the extent and appearance of soft tissue and since MRI gives the best (anatomical) soft tissue contrast, currently tumour staging is best done using MRI to assess the extent of the disease local to the large bowel. The primary tumour site is assessed to determine if the disease has extended into the adjacent fat 


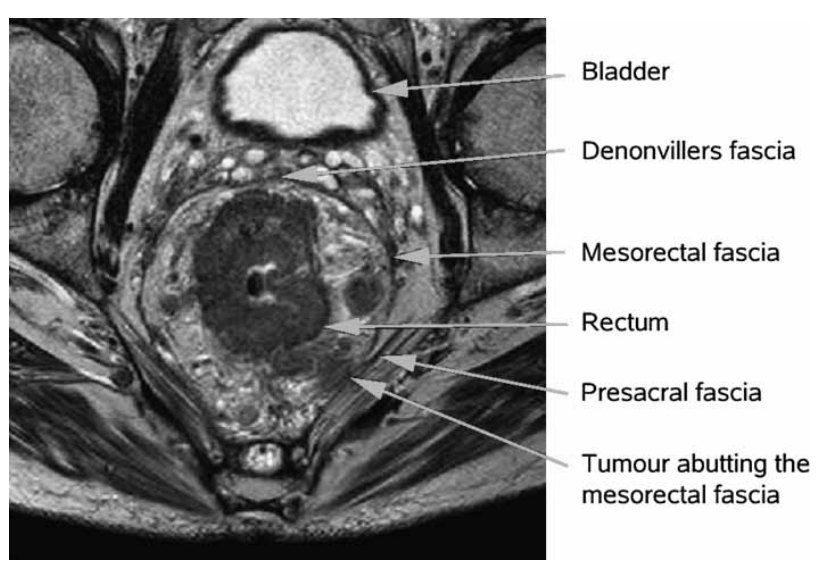

Figure 1. Axial T2-weighted fast-spin echo image showing a tumour of the mid-rectum. There is a focus of tumour abutting the mesorectal fascia. Primary surgery in this patient would result in a positive surgical circumferential resection margin.

and invaded the adjacent tissue planes, including the mesorectum. As indicated by the survival rates above, a close proximity between the primary tumour site and the wall of the mesorectum gives a high probability of the tumour being incompletely resected and an associated increased risk of metastatic disease. This has led to the suggestion that an important predictor of likely success of total mesorectal resection is the circumferential resection margin, the distance of the closest affected sentinel lymph node to the surface of the mesorectal fascia. Similarly, an infiltration of the lymph nodes by the tumour is a predictor of metastasis. It is therefore important, in examining the MRI data (or data from another modality), to be able to detect not only the geometry of the primary tumour, but also other key features and boundaries. An annotated MRI slice showing the presence of a tumour in close proximity to the mesorectal fascia is shown in figure 1 .

\section{The data}

Research conducted within the magnetic resonance imaging and rectal cancer equivalence study (MERCURY) project $\dagger$, which involved over 20 clinical centres, presents strong evidence that the use of MRI can be an effective predictor of the success of resection in patients with colorectal cancer. We believe that by improving the interpretation of MRIs as well as by giving a more quantitative measure of surgical quality, patient prognosis can be further improved. Furthermore, by linking different image types, communication between radiologists and pathologists may be enhanced.

As part of the MERCURY project, the Royal Marsden Hospital, London has collected MRI volumes of colorectal tumours from numerous locations. The MRI volumes were obtained prior to chemo/radiotherapy and prior to surgery. They are anonymized and each case has its own MERCURY identifier. These volumes are also accompanied with descriptive

$\dagger$ The MERCURY project, http://www.pelicancentre.com/researchprojects/mercury.html. 

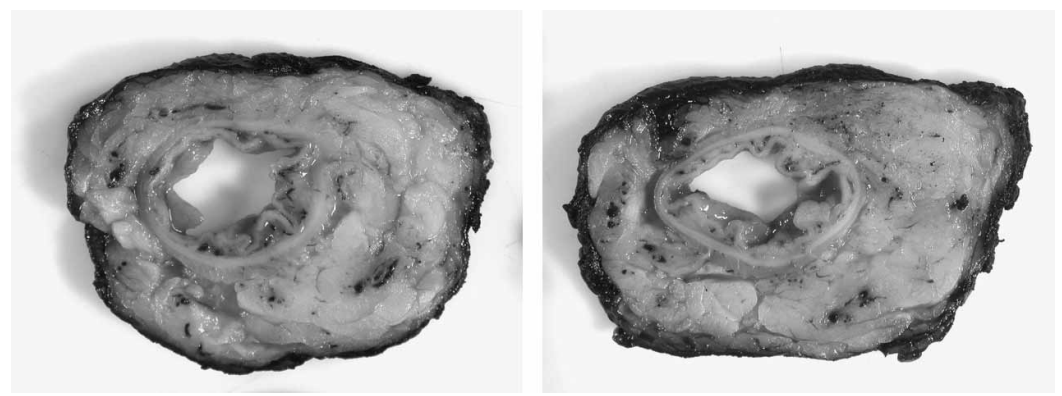

Figure 2. Two adjacent macroscopic slices from the Leeds Virtual Pathology data†. Note that the mesorectum is yellow.

meta-data such as the MRI position, the extent of the tumour and the surgical plan. An example slice from an MRI volume is shown in figure 1.

The other data resource that our current project draws upon is a collection of macroscopic and microscopic slides in digital format, which were scanned using ScanScope ${ }^{\mathrm{TM}}$ scanners at Leeds University. The ScanScope ${ }^{\mathrm{TM}}$ scanner generates TIFF data that is immediately viewable using Aperio's ImageScope ${ }^{\mathrm{TM}}$ viewer. This same software can also be used to annotate the micro-images in order to produce annotated micro-data stored in an XML format. Currently, each micro-image is stored in a custom file format (SVS) created by the scanner and displayed only in its associated viewer. Examples of macroscopic and microscopic data are shown in figures 2 and 3, respectively.

\section{Image processing and data registration}

This section deals with the in silico construction of 3D geometric models of the large bowel from MRI, macroscopic and microscopic data. Currently our work is focused on accurately representing the geometry of a resected tissue sample after it has been sliced and photographed. Future work will include the automatic detection of tumours and the integration of data from different modalities.

\subsection{Segmentation}

The first step in the process of constructing a geometric model from macroscopic histopathology slices is segmentation which identifies areas of the image that belong to different tissue types. Often, in this case, segmentation can be a simple thresholding process, since, unlike normal grey-scale MRI images, pathology images are coloured and the colour bands give important information about tissue type. For instance, the mesorectal fascia appears more yellow than the rectum (and tumour) in the macroscopic slices, as can be seen in figure 2. We convert the images from RGB colour mode to CMYK colour mode so that we can threshold in the yellow channel to distinguish the rectum from the mesorectal fascia.

$†$ Virtual pathology at the University of Leeds, http://www.virtualpathology.leeds.ac.uk/. 


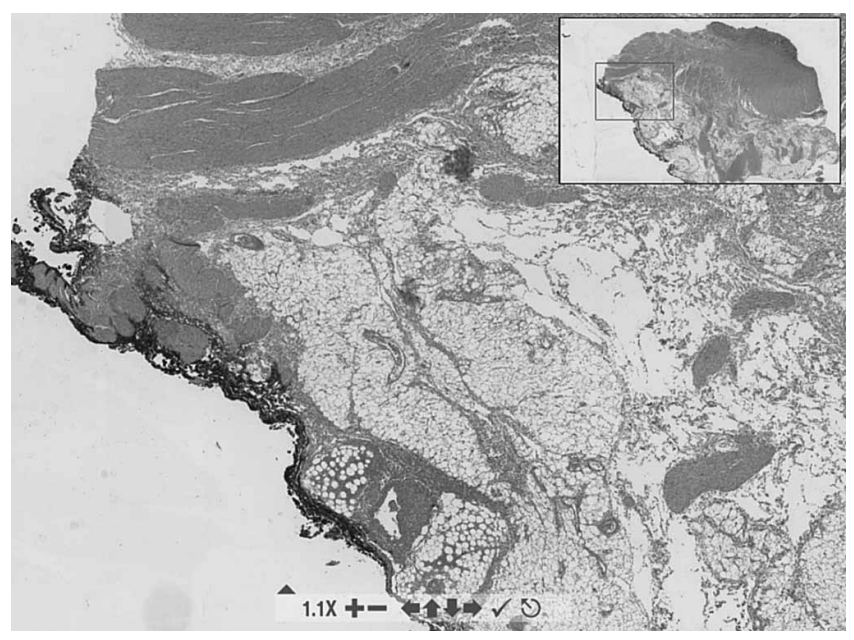

Figure 3. High-resolution microscopic slice from Leeds Virtual Pathology $\dagger$ explored within an SVS image viewer.

The results of segmentation are represented as contours that correspond to the boundaries between the segmented regions. In practice, the initial contours produced by thresholding often contain intrusions and extrusions. We know, however, that the contours of rectum walls are approximately circular in shape, so we apply a series of standard morphological operations to remove the unwanted intrusions and extrusions.

\subsection{Registration}

Pair-wise registration is required for macroscopic slices because tears, fissures, twists and other deformations can occur during the cutting process and/or as the consequence of gravity when the slices are put down to be photographed. The distance between two adjacent slices is usually sufficiently large ( 3 or $7 \mathrm{~mm}$ ) to rule out correspondences between slices based on texture, so we use a contour based registration method: we find the centre of area of the stable feature (the rectum wall) and use it as the pseudo-centre for alignment. The orientation of each slice is then estimated by maximising the overlapped area with the last adjacent slice. This process of identifying pseudo-centres and orientations is iterated until a maximum overlapped area is found. Currently, macroscopic slices are transformed rigidly and put into a $3 \mathrm{D}$ volume.

It should be noted that contour-based registration relies on segmentation being accurate. When the similarity, which we measure as the percentage of the overlapped area relative to the whole area, between the registered pair is below a certain value, the registration may converge to an unwanted local minimum. This can be caused by the difference between slices and/or by unsatisfactory segmentation. In the latter case, human intervention is required to digitise the contours manually.

Elastic registration has yet to be implemented. We plan to use contour matching techniques to find a displacement field for the pixels on the contour, which will be followed by the interpolation of the displacements of regions bounded by contours. We plan also to implement simultaneous segmentation and registration, within an expectation-maximisation framework. 
The techniques involved in MRI volume reconstruction are more straightforward, since the volume is presented in one contiguous piece (albeit digitised into voxel slices) without any additional tissue damage. The MRI segmentation method uses an active contour (snake) to detect the rectum contour, because the rectum is always closed in vivo. The processing of the resected post-surgery MRI of the specimen is similar.

\subsection{Combining modalities}

The process of combining data from different modalities is an example of 3D registration. This has not yet been implemented, but the sequence of computational operations is understood. In our case, a series of pre-surgery MRI slices will first be segmented and then put into a 3D volume according to the (recorded) angles of the slices. After resection, the macroscopic photographs of slices will be combined to produce another 3D volume. These two volumes will be registered with each other, for example by considering points of high mutual information. This registration is more complicated than the $2 \mathrm{D}$ registration because of an increase in the dimension, because of an increase in the number of potential sites of mutual information and because rigid-body registration provides a poor model. The shape of the colon in vivo differs from its shape after resection and slicing when it is no longer surrounded by supporting tissue. Consequently, a non-rigid elastic model will have to be incorporated into the $3 \mathrm{D}$ registration process.

Once two or more modalities have been registered, it is possible to present data from one modality in the context of another. So, for example, a clinician could explore a pre-therapy MRI volume of the colon and request that the histopathological data to be overlayed on the volume. This would give the cancer specialist a means of assessing and quantifying the exact changes in tumour size before therapy and after resection.

The most informative way to present different imaging modalities with their various orientations in a single volume is, as yet, unclear. However, by providing clinicians with various options and then discussing their usefulness, we hope to progress towards a pragmatic user interface design. As a first iteration in this process, we have produced a simple demonstration of the volume reconstruction procedure. A program, written in $\mathrm{C}$ using OpenGL, takes as input a series of JPEG macroscopic images. It also reads an XML description (from the output of the registration program) of the two identified contours and the relative orientation after registration for each image. The registration is performed using a rigid-body model on the outer mesorectum contour. The reconstruction of the image stack into a 3D volume of the colon is then animated (see figure 4), so that the user can see how the images align within the stack and can see the contours that were identified.

\section{Clinical and modelling uses for the data}

The ultimate goal of mathematical modelling of cancer is to develop in silico virtual tumours which can be used to generate experimentally testable hypotheses concerning the initiation, development, growth and spread of tumours and the likely efficacy of any proposed drugs and/or treatment regimes for individual patients. Simply by looking through the different papers within this special issue, we can perhaps begin to imagine how such a mathematical model might look: a hybrid approach that couples, as appropriate, stochastic or deterministic approaches with techniques drawn from the array of mathematical tools currently available to the systems biologist. 


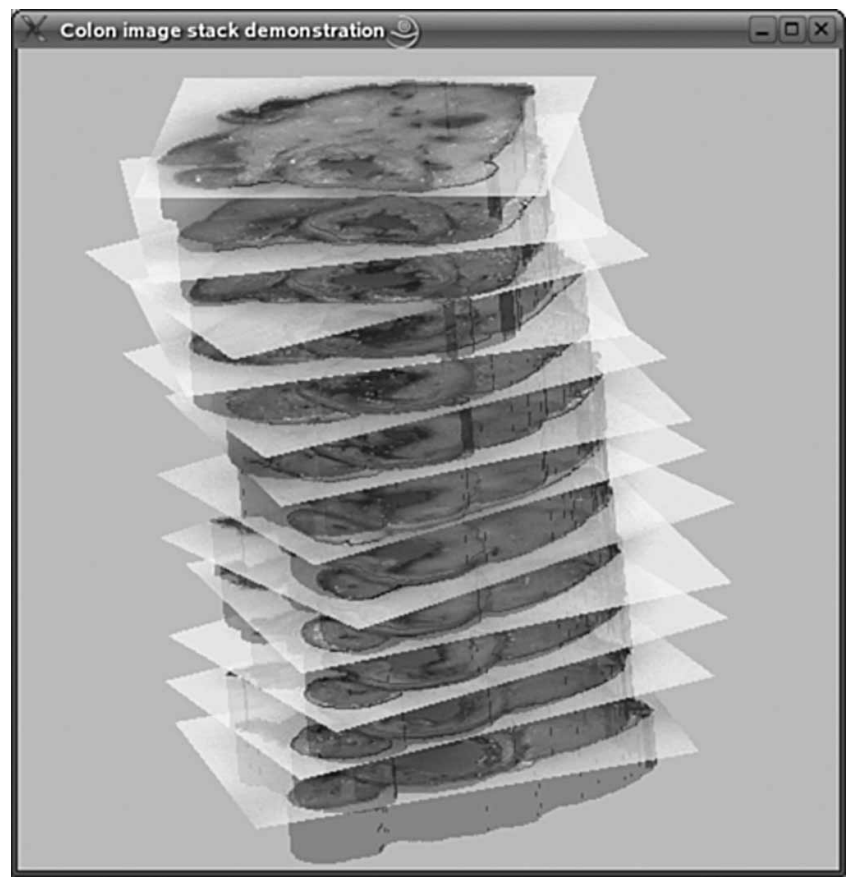

Figure 4. Simple OpenGL reconstruction from macroscopic slices. The inclusion of the full photographs shows their relative orientations before registration. The contours of the outer mesorectum and of the bowel wall are marked in blue and red respectively.

\subsection{The integrative biology project}

A multi-scale model for colorectal cancer is being developed within the framework of the international IB e-science project $\dagger$, which brings together computer scientists, modellers and experimental researchers [5]. Existing models for colorectal cancer are largely very simple and generally focus on a single time and length scale (for a review, see [6]). The IB-approach, instead, integrates descriptions of all levels of organisation from molecule to tissue. The multi-scale model can be classified as a hybrid cellular automaton. At the subcellular level, deterministic continuum models are used to characterise biochemical networks, including Wnt signalling and cell-cycle control. The outputs of such subcellular models regulate the behaviour of the monolayer of epithelial cells that covers the luminal surface of the intestine and lines the intestinal crypts. In particular, the presence of Wnt gradients along the crypt axis determines the spatially dependent rates of cell proliferation, differentiation, apoptosis and migration. Under normal conditions, these cellular processes are tightly coordinated and the number of cells remains approximately constant, whereas under aberrant conditions their dysregulation causes increased proliferation (and/or decreased differentiation). The resulting proliferative excess has serious implications for the maintenance of the integrity of the crypt, as it induces biomechanical stress that can force the crypt to deform and undergo fission to accommodate the newborn cells. In most cases, these phenomena at the tissue level lead eventually to polyp formation. Integrating the hierarchy of processes occurring at different

$\dagger$ The Integrative Biology project, http://www.integrativebiology.ox.ac.uk/. 
levels of organisation into a multi-scale model, the IB team aims to be able to investigate possible interactions between such processes, to combine biochemical, histological and clinical data, and to test drugs in silico on the system as a whole.

\subsection{Use of imaging techniques within mathematical models}

It is key to the success of mathematical modelling of cancer to be able to extract appropriate and accurate parameter values from both in vitro and in vivo systems and to devise methods to validate the models against experimental data. To do this in human subjects requires that it be done in a minimally invasive manner, hence the use of state-of-the-art imaging technologies will be crucial.

We are exploring several avenues to this end. At the radiological scale $(\approx 1 \mathrm{~mm})$, we are exploring the application of MRI to model the changes in shape and heterogeneity over time of a developing tumour, applying novel $\mathrm{pH}$ imaging technologies in MRI to validate models of tumour acidosis [7] and using PET to analyse and simulate both tumour hypoxia [8,9] and response to radio-labelled drugs $[10,11]$. At the same time, we are beginning to explore the application of so-called molecular imaging techniques [12] to explore tumour development at the $\approx 1 \mu \mathrm{m}$ scale.

\subsection{Potential use-case}

It is beyond the scope of the current paper to discuss in detail all of the possible applications of imaging technologies in the parameterisation and validation of mathematical approaches. Instead, we illustrate the potential of these technologies focusing on a single scenario within the field of colorectal cancer. In surgical planning, a subjective judgement must be made that balances the competing risks of removing too little or too much tissue from a cancerous region. One goal of our mathematical modelling is to develop a realistic model of the growth of a tumour in situ in a heterogeneous medium. To do this, we will need a way of validating the predicted growth at both the micro-scale, in terms of individual cells and at the tissue level, in terms of the morphology and the overall geometry of the cancerous region. This validation will require the extraction of physiological parameters from histology images at the cellular level and both physiological and geometric information from MRI images at the

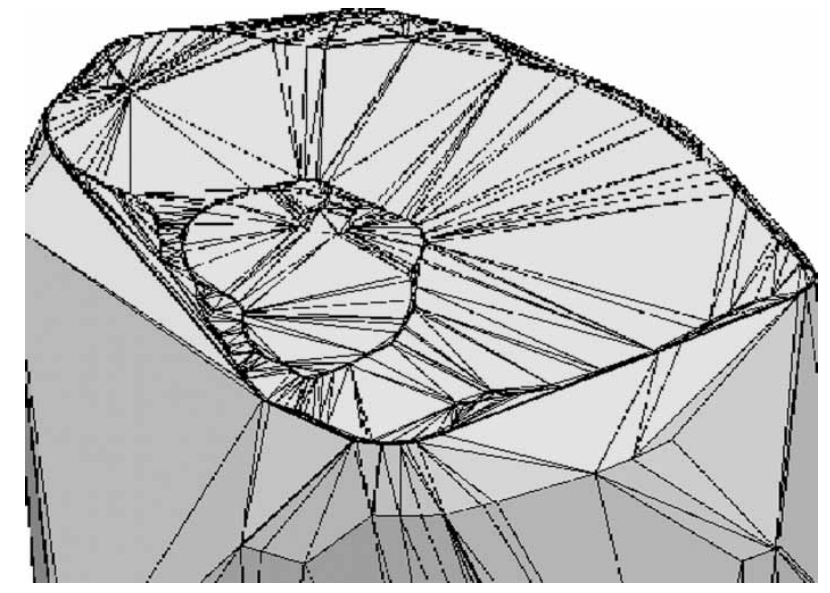

Figure 5. Delaunay mesh based on the convex hull of the reconstruction from macroscopic slices. 
tissue level and to be able to make use of the techniques described in Section 4 to fuse this information together.

In figure 5, we give an example of just one step in the process explained above. We extracted the geometry of a resected portion from a human colon by using the points on the mesorectum and bowel wall contours after registration to seed a 3D mesh. This generated a Delaunay tetrahedral mesh of the convex hull of the sample, which is focused towards the original contours. With some pre-processing effort, it is possible to use the same contour data to produce a more uniform mesh in which segmentation results are encoded (i.e. with elements representing colon, mesorectum, tumour and air). Similar techniques can be applied to the in vivo MRI image data both pre- and post-resection. The resulting geometries can then be compared and used as a tool by surgeons wanting to assess the extent to which the actual resection matched the initial surgical plans.

It is also possible to envisage how this geometric information can be combined with the estimation of physiological parameter values using in vivo MRI and PET imaging data to develop in silico models of patient-specific tumour growth dynamics to predict the future behaviour of the neoplasm or to reconstruct the past history of the disease for use in surgical planning. For example, the geometric information from MRI data could be used to generate a mesh to be used in finite element modelling, with the parameter values of the underlying system of partial differential equations estimated, perhaps automatically, from the image data. A surgeon could then explore in silico the likely effects of key parameters within the model (for example, those governing the aggressiveness of the tumour) and in particular, visualise these effects within the multi-model image stack, which would provide a valuable tool in surgical planning.

This initial use case covers just two of the levels (cellular and tissue) described by Bellomo and Maini [4]. It is not difficult to envisage, however, how processes at the sub-cellular level could also be incorporated through coupling of models describing molecular processes. Those molecular processes themselves could in turn be parameterised via molecular imaging techniques, as recently demonstrated by one of Maini's co-workers [13].

\section{Architecture for the secure aggregation of data}

The main technological goal of our current project is to develop a prototype tool to integrate and display pathology and radiology images (MRI volume prior to chemotherapy and surgery) of a case having colorectal cancer by using existing grid technology at the database federation level, together with an interface to a collection of image analysis tools to meet the application goals of the project described above.

To achieve our technological goal, it is essential to verify that both MRI and pathology images come from the same patient cases and that these two sets of data can be related (in both technical and ethical senses). If this is the case, we can then relate and integrate pathology and radiology images of a case in two ways. The first way to link the different types of data involves the integration of the full images as entities; this means that an available pathology image may be retrieved together with its associated MRI image/report. The second method is via the association of the corresponding region of interest (ROI), i.e. if we have a ROI in an MRI volume, then we should be able to retrieve its associated area from the 3D pathology images.

Often each MRI volume has more than one ROI, because individual lymph nodes could also be annotated as well as the main tumour, although only one of these annotations would 
apply to a lesion. Thus, a flexible database schema that can effectively describe all relevant data is essential. It is also important that certain commonly computed data (such a contour identification and relative orientation after registration) can be tagged to the main data and written back into the database at some level. All the data is stored in a federated database that is distributed over several sites, with all of the data servers accessible via web service interfaces.

The infrastructure that is underpinning the demonstrator is based on the output of the eDiaMoND project [14] and is a realisation of the architecture of Ref. [15]. The patient and image data are stored at the site at which they originated, with the data from each location reflecting the specialisation of that site. The selection of MRI and pathology data pertaining to a single case is achieved by aggregating data from multiple sites.

A user at a workstation requests access to certain data. This request is sent to an externally facing service that, if necessary, sends the request to other sites via communication with those other sites' externally facing services. The relevant external services then pass appropriate requests to their corresponding internal services, which then use the local access control policy to decide if the user has the necessary access. If access is granted, the requested data is then returned to the querying user.

Once the data acquisition requirement has been met, it is then necessary for the system to facilitate the remote execution of various software components, such as 3D reconstruction algorithms. Initially, this is being achieved by the redeployment of technology developed within eDiaMoND.

A number of functions are provided in the form of an application programmer's interface (API). These functions include methods of retrieving data based on selection criteria with the intention being that this API is used by imaging tools that interface with the underlying databases. The utilisation of the API effectively hides from the imaging application the fact that it is dealing with a distributed federated data source: as far as the application is concerned, it is dealing with a single, logical database.

Experience gained in previous projects, including eDiaMoND and NeuroGrid [16] has informed the technical decisions made with respect to the underlying infrastructure. In particular, this experience has allowed us to develop an architecture that provides secure access to a virtual data source made up from data held on distributed servers.

Access to the data is restricted to project partners by means of certificate-based security mechanisms. The servers authenticate the certificate passed by the requester and the requester checks that the certificate from the server is one that is trusted. This then allows a signed and encrypted data channel to be opened between the requester and server. The signature part of the process provides assurance that the data received was the data that was sent. This is of particular importance in the case of image data where corruption to very small areas leads to significant errors in image processing operations.

\section{Conclusion}

In this paper, we have presented our initial work in developing a multimodal, multi-scale approach to the analysis of images of colorectal cancer. Among the various potential clinical applications of our work, we have chosen to focus on the parametrisation and validation of multiscale mathematical models. Although in this paper, we have described the application of these techniques to colorectal cancer only, we believe that, as the "systems" approach to biological and physiological modelling takes hold, the use of image and signal analysis techniques will become increasingly important within the clinical domain. The development of fully automated image analysis tools, such as those described here, will greatly facilitate this process. 


\section{Acknowledgements}

This research was supported by the Medical Research Council, The Wellcome Trust, the Department of Health, IBM and the EPSRC (via the Integrative Biology project, GR/S72023).

\section{References}

[1] Araujo, R.P. and McElwain, D.L.S., 2004, A history of the study of solid tumour growth: the contribution of mathematical modelling, Bulletin of Mathematical Biology, 66, 1039-1091 September.

[2] Roose, T., Chapman, S.J. and Maini, P.K., 2006, Mathematical models of avascular tumour growth, SIAM Review.

[3] Byrne, H.M., Alarcon, T., Owen, M.R., Webb, S.D. and Maini, P.K., 2006, Modelling aspects of cancer dynamics: a review, Philosophical Transactions of the Royal Society A, 364(1843), 1563-1578.

[4] Bellomo, N. and Maini, P.K., 2005, Special issue on cancer modeling-preface, Mathematical Models and Methods in Applied Sciences, 15(11), iii-viii.

[5] Gavaghan, D.J., Simpson, A.C., Lloyd, S., Mac Randal, D.F. and Boyd, D.R., 2005, Towards a grid infrastructure to support integrative approaches to biological research, Philosophical Transactions of the Royal Society A, 363(1833), 1829-1841.

[6] van Leeuwen, I.M.M., Byrne, H.M., Jensen, O.E. and King, J.R., 2006, Crypt dynamics and colorectal cancer: advances in mathematical modelling, Cell Proliferation, 39(3), 157-181.

[7] Smallbone, K., Gavaghan, D.J., Gatenby, R.A. and Maini, P.K., 2005, The role of acidity in solid tumour growth and invasion, Journal of Theoretical Biology, 235(4), 476-484 Aug.

[8] White, C.J. and Brady, J.M., A model to simulate tumour oxygenation and dynamic [18F]-Fmiso PET data, Physics in Medicine and Biology, to appear.

[9] White, C.J. and Brady, J.M., 2006, Hypoxia: from modelling molecules to molecular imaging. Proc. Society of Nuclear Medicine Annual Meeting, June.

[10] Ali, R., Schottlander, D. and Brady, J.M., 2006, Development and validation of a simulated human torso dynamic FHBG-PET dataset. American Molecular Imaging Conference, March.

[11] Ali, R., Schottlander, D., Yau, C. and Brady, J.M., 2006, Sensitivity analysis of a three compartment model used in PET tracer kinetic modelling. American Molecular Imaging Conference, March.

[12] Masoud, T.F. and Gambhir, S.S., 2003, Molecular imaging in living subjects: seeing fundamental biological processes in a new light, Genes and Development, 17, 545-580.

[13] Smallbone, K., 2006, The role of acidity in tumour development, PhD thesis, University of Oxford.

[14] Brady, M., Gavaghan, D., Simpson, A., Highnam, R. and Mulet, M., 2003, eDiamond: a grid-enabled federated database of annotated mammograms, Chapter 39. Grid Computing: Making the Global Infrastructure a Reality (New York: John Wiley and Sons, Ltd.).

[15] Power, D.J., Politou, E.A., Slaymaker, M.A. and Simpson, A.C., 2005, Towards secure grid-enabled healthcare, Software: Practice and Experience, 35(9), 857-871.

[16] Geddes, J., Lloyd, S., Simpson, A., Rossor, M., Fox, N.C., Hill, D.L.G., Hajnal, J.V., Lawrie, S., McIntosh, A., Johnstone, E., Wardlaw, J., Perry, D., Procter, R., Bath, P. and Bullmore, E.T., 2005, Neurogrid: using grid technology to advance neuroscience. 18th IEEE Symposium on Computer-Based Medical Systems (CBMS 2005) 23-24 June, (Dublin, Ireland) pp. 570-572. 


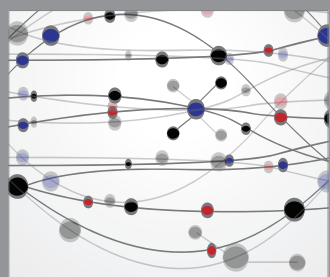

The Scientific World Journal
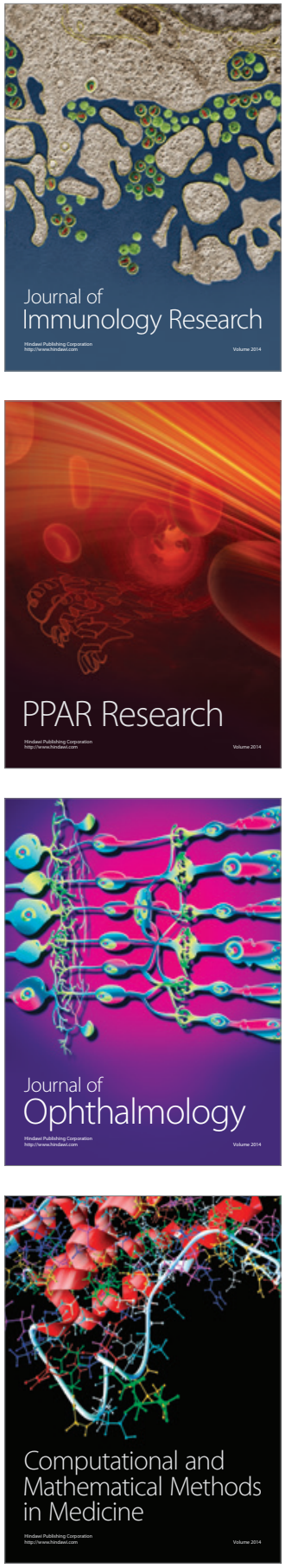

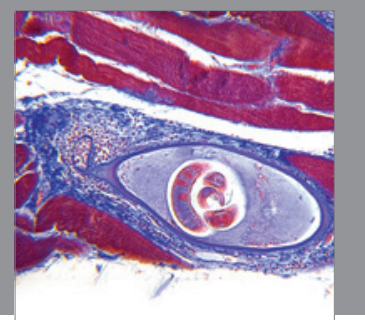

Gastroenterology

Research and Practice
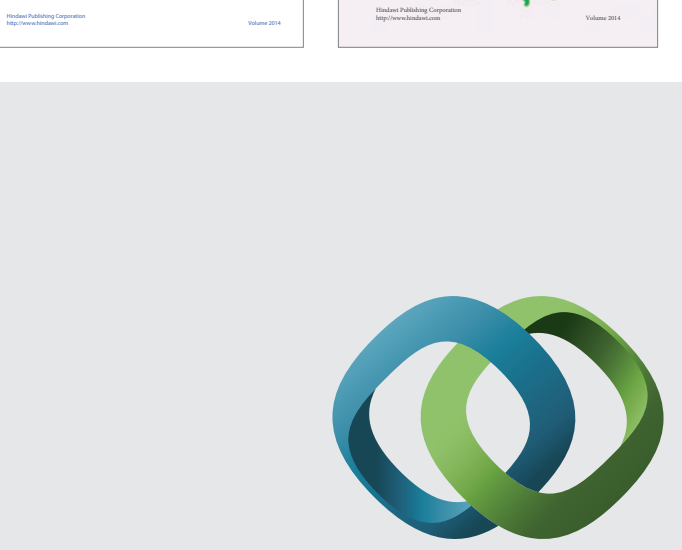

\section{Hindawi}

Submit your manuscripts at

http://www.hindawi.com
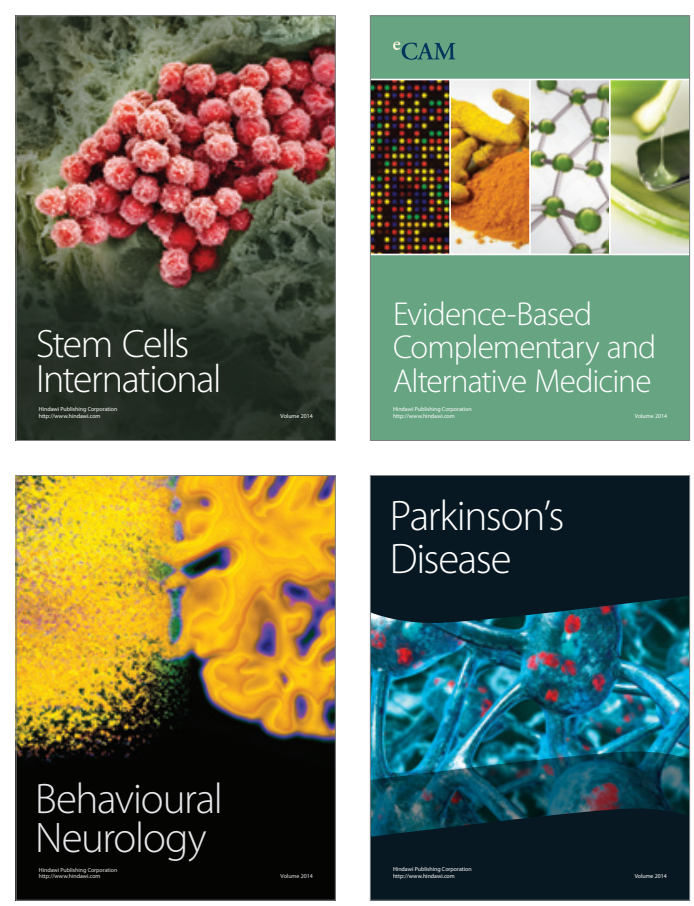

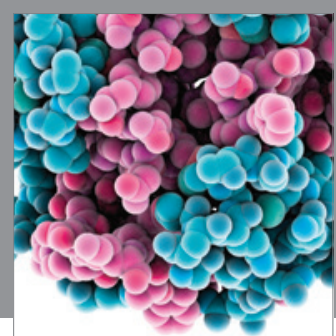

Journal of
Diabetes Research

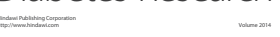

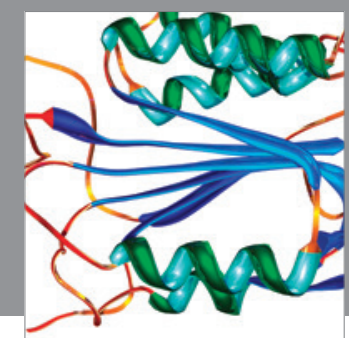

Disease Markers
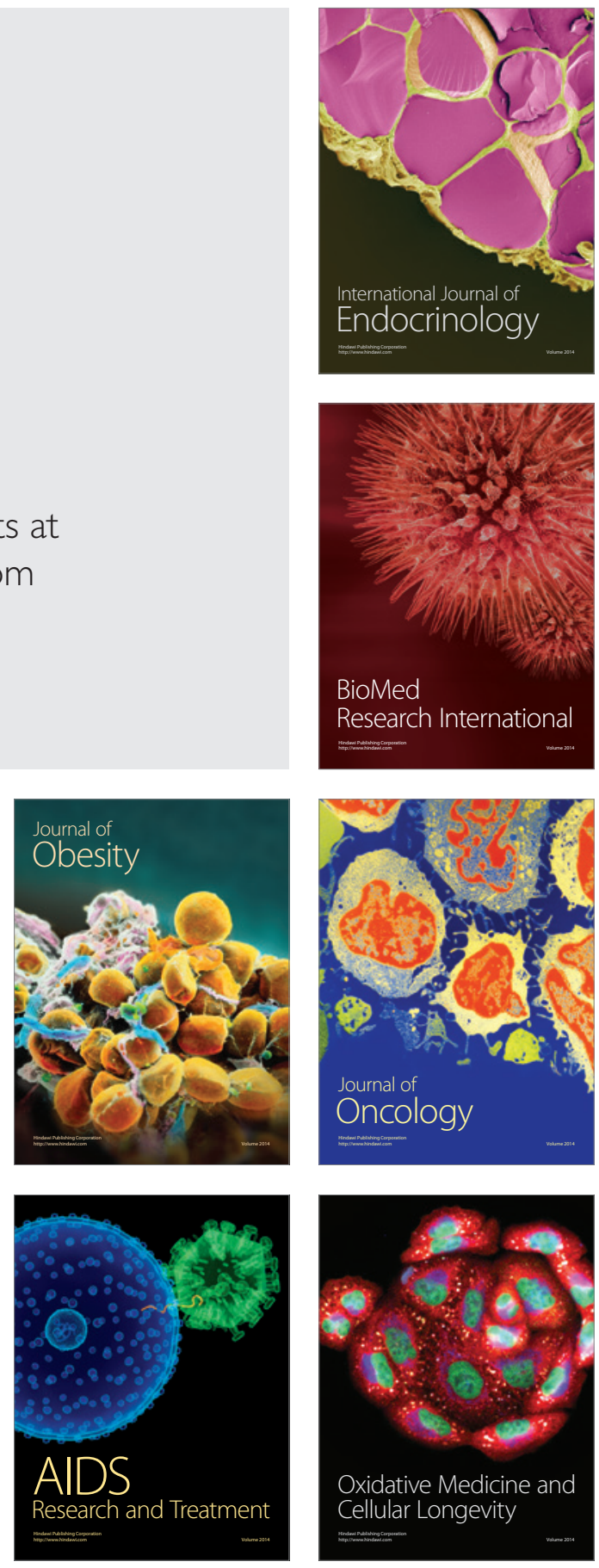